9 - ORIGINAL ARTICLE

ISCHEMIA-REPERFUSION

\title{
Importance of duration and number of ischemic postconditioning cycles in preventing reperfusion mesenteric injuries. Experimental study in rats ${ }^{1}$
}

\author{
Carlos Henrique Marques dos Santos ${ }^{\mathrm{I}}$, Ricardo Dutra Aydos ${ }^{1}$, Ed Nogueira Neto ${ }^{\text {II }}$, Luciana Nakao Odashiro Miiji ${ }^{I I}$, Pedro \\ Carvalho Cassino ${ }^{\mathrm{IV}}$, Isadora Ishaq Ahmed ${ }^{\mathrm{v}}$, Nadia Meneguesso Calheiros ${ }^{\mathrm{v}}$, Milena Garciav, Anderson Fernandes da Silva ${ }^{\mathrm{IV}}$
}

DOI: http://dx.doi.org/10.1590/S0102-865020150100000009

IPhD, Associate Professor, Department of Surgery, Universidade Federal do Mato Grosso do Sul (UFMS), Campo Grande-MS, Brazil. Conception and design of the study, interpretation of data, manuscript writing, critical revision.

IIMD, Resident, Department of Surgery, UFMS, Campo Grande-MS, Brazil. Acquisition, interpretation and analysis of data.

IIIPhD, Associate Professor, Department of Pathology, UFMS, Campo Grande-MS, Brazil. Histopathological examinations.

${ }^{\text {IV } M a s t e r, ~ F e l l o w ~ P h D ~ d e g r e e, ~ P o s t g r a d u a t e ~ P r o g r a m ~ i n ~ H e a l t h ~ a n d ~ D e v e l o p m e n t ~ i n ~ R e g i o n ~ M i d w e s t, ~ U F M S, ~ C a m p o ~ G r a n d e-M S, ~ B r a z i l . ~ T e c h n i c a l ~}$ procedures, acquisition of data.

${ }^{\mathrm{v}}$ Graduate student, School of Medicine, UFMS, Campo Grande-MS, Brazil. Acquisition, interpretation and analysis of data.

\begin{abstract}
PURPOSE: To evaluate the effect of ischemic postconditioning (IPC) on intestinal mucosa of rats subjected to ischemia and reperfusion process comparing two cycles of reperfusion and ischemia lasting two minutes each and four cycles of reperfusion and ischemia lasting 30 seconds each

METHODS: Thirty Wistar rats were distributed into three groups: group A (10 rats), ischemia (30 minutes) and reperfusion (60 minutes); group B (10 rats), ischemia and reperfusion plus IPC by two lasting two minutes each; and Group C (10 rats), ischemia and reperfusion plus IPC by four cycles lasting 30 seconds each. Finally, a segment of small intestine was resected for histological analysis. We analysed the results according to Chiu et al. classification and proceeded to the statistical treatment by Kruskal-Wallis test ( $\mathrm{p}<0.05$ ). RESULTS: The mean degree of tissue injury according to Chiu et al. classification were: Group A, 2.77; in group B, 1.4; and group C, 1.4. B X C $(\mathrm{p}<0.05)$.

CONCLUSIONS: Ischemic postconditioning was able to minimize reperfusion injury of rats undergone mesenteric ischemia and reperfusion process. There was no difference in the effectiveness of the method comparing two cycles of two minutes with four cycles of 30 seconds by H\&E histological evaluation of the ileum after 60-minute reperfusion.
\end{abstract}

Key words: Ischemic Postconditioning. Ischemia. Reperfusion. Intestines. Rats. 


\section{Introduction}

Since 1986, when Parks and Granger ${ }^{1}$ demonstrated the harmful effects of toxic reactive oxygen species (ROS) produced during reperfusion, many researches have been developed in search of an experimental model that could minimize this process in order to reduce the cellular and organic damage of ischemia and reperfusion ${ }^{2,3}$.

The best results ever published in controlling the production of ROS were obtained with the ischemic preconditioning, as numerous publications that followed Murry et $a l .{ }^{4}$, including the mesenteric ischemia and reperfusion. However, it has little clinical applicability in situations like, for example, acute abdomen with mesenteric vascular ischemia, therefore, at the time of diagnosis, the ischemia is already installed.

Besides the acute mesenteric ischemia, some intestinal surgery, especially resection and transplantation, are performed through temporary occlusion of mesenteric vessels to prevent bleeding, which may occur in such situations of reperfusion injury.

Zhao et $a .^{2}$ presented the concept of ischemic postconditioning (IPC), which consists of making one or more short cycles of reperfusion followed by one or more short cycles of ischemia, immediately after ischemia and before permanent reperfusion.

In experimental model, there are already evidence of the protective effect of IPC on the intestinal mucosa of rats subjected to mesenteric ischemia and reperfusion ${ }^{5}$, and, recently, the IPC was able to minimize the severity of liver injury in rats subjected to ischemia and reperfusion ${ }^{6}$ for through three cycles of ischemia and reperfusion for two minutes.

Several published experiments examined the effects of IPC in other organs and tissues, among which may be mentioned Darling et al. ${ }^{7}$, in which the IPC was able to minimize the area of infarction in myocardium of rabbits; Tang et al. ${ }^{8}$ in which IPC demonstrated efficacy in preventing injuries resulting from the process of coronary ischemia and reperfusion in rats, since the ischemia time did not exceed 45 minutes; Huang et al. ${ }^{3}$ demonstrated that with IPC was preventing tissue damage in the spinal cord of rats subjected to ischemia and reperfusion; Santos et $a l .{ }^{9}$ (three cycles R/I during two minutes) showed that the IPC was able to minimize the tissue injury in the intestinal mucosa of rats undergone mesenteric ischemia and reperfusion process.

Many other authors have demonstrated the efficacy of PCI in mesenteric I/R, but with too variated methods. Sengul et $a l .{ }^{10}$ (six cycles R/I during 10 seconds), Cheng et al. ${ }^{11}$ (three cycles
$\mathrm{R} / \mathrm{I}$ during 30 seconds), Wen et al. ${ }^{12}$ (three cycles R/I during 30 seconds), Onody et al. ${ }^{13}$ (six cycles R/I during 10 seconds) and Rosero et $a l .{ }^{14}$ (three cycles R/I during 60 seconds and six cycles of R/I during 10 seconds) have founded good results with PCI but, how we can see, with great variation in methods.

Moreover, Bretz et al. ${ }^{15}$ published a study in rabbits showing that the IPC performed with four cycles of 30 seconds of reperfusion and 30 seconds of reocclusion did not have statistical significance on the degree of necrosis of the intestinal mucosa. Also showed no benefit in the use of IPC in intestinal ischemia and reperfusion Nakamura et al. ${ }^{16}$, using five cycles of reperfusion and ischemia of 30 seconds each.

Thus, although there are much evidence of the effectiveness of IPC, it is not yet determined which would be the best method of developing it, how many cycles, the duration of each cycle, if there are differences when used for bowel or other organs, etc.

Then, considering the current evidence of the value of IPC in minimizing tissue damage resulting from ischemia and reperfusion, it is of fundamental importance, and the aim of this study, to evaluate the effectiveness of IPC comparing two different methods on the number and duration of the cycles.

\section{Methods}

The study was approved by the Ethics Committee for Animal Experimentation of the Universidade Federal do Mato Grosso do Sul (594/2014) and was based on ethical principles advocated by the Brazilian College of Animal Experimentation.

We used 30 rats (Rattus norvegicus) of the Wistar albino strain adult male, weighing 270-350 grams, with an average of 305 grams, from the vivarium of the Federal University of Mato Grosso do Sul. The animals were distributed in following groups (Figure 1):

Group A - Ischemia and Reperfusion: Ten rats undergone intestinal ischemia for 30 minutes by occlusion of the cranial mesenteric artery with a vascular clamp, followed by reperfusion for 60 minutes for removal of the clamp.

Group B - Ischemic postconditioning 1 (IPC-1): Ten rats undergone ischemia for 30 minutes by occlusion of the cranial mesenteric artery with vascular clamp and reperfusion for 60 minutes. Between ischemia and reperfusion two reperfusion cycles (two minutes each) were performed, interleaved by two ischemia cycles (two minutes each).

Group C - Ischemic postconditioning 2 (IPC-2): Ten rats undergone ischemia for 30 minutes by occlusion of the 
cranial mesenteric artery with vascular clamp and reperfusion for 60 minutes. Between ischemia and reperfusion four cycles of reperfusion (30 seconds each) were performed, interleaved by four cycles of ischemia (30 seconds each).

\section{Group A}
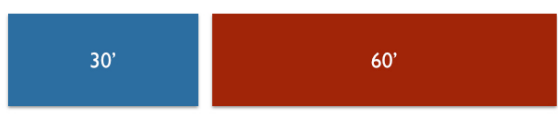

\section{Group B}

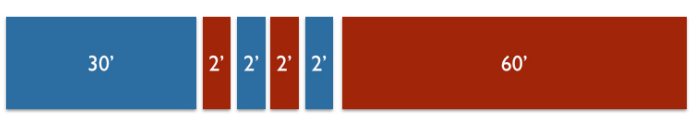

Group C

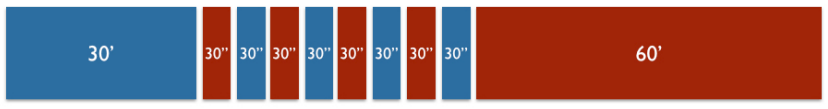

FIGURE 1 - Schematic demonstration of periods of ischemia and reperfusion in groups $\mathrm{A}, \mathrm{B}$ and $\mathrm{C}$ (the numbers corresponds to time in minutes; blue: ischemia; red: reperfusion).

The animals were anesthetized by intraperitoneal injection of 2:1 solution of Ketamine $\left(\operatorname{Cetamin}^{\circledR}\right), 50 \mathrm{mg} / \mathrm{ml}$, and Xylazine $\left(\right.$ Xilazin $\left.^{\circledR}\right), 20 \mathrm{mg} / \mathrm{ml}$, respectively, at a dose of $0.1 \mathrm{ml} / 100 \mathrm{~g}$. The rats were considered anesthetized after being found loss of eyelid reflex, loss of response to mechanical stimuli, loss of righting reflex and withdrawing member after painful stimulus caused by hold.

After anesthetized rats were subjected to abdominal trichotomy and positioned to the operating table in the supine position with the four members in abduction. Then a longitudinal median laparotomy of about four centimeters was performed, followed by exteriorization of the small intestine, identification and dissection of the mesenteric artery.

In group $\mathrm{A}$, the mesenteric artery was occluded with atraumatic vascular clamp which remained for thirty minutes (ischemic phase). After placing the clamp, the small intestine was repositioned into the abdominal cavity and the wound was closed with a continuous suture of the skin with nylon monofilament (mononylon $^{\circledR}$ ) 4-0. After the stage of ischemia, the abdominal wall was opened again by removing the suture and the vascular clamp was removed, beginning the reperfusion phase, lasting 60 minutes. Started the reperfusion, the abdomen was closed again by continuous suture until the end of the experiment.

In group $\mathrm{B}$, after ischemia phase (30 minutes) was performed the IPC through two cycles of reperfusion, lasting two minutes each, interspersed by two cycles of ischemia also lasting two minutes each. After that, it was performed reperfusion for 60 minutes.

In group $\mathrm{C}$ was performed ischemia phase (30 minutes) and reperfusion (60 minutes). Preceding the reperfusion IPC was performed through four cycles of reperfusion lasting 30 seconds each, interspersed by four cycles of ischemia also lasting 30 seconds each.

After completion of reperfusion, in the three groups, the abdominal wall was opened again by removing the suture and a segment of approximately 1-cm-longer ileum was resected five centimeters proximally to the ileocecal transition, which was opened on its antimesenteric edge, washed with saline solution, and then placed in a $10 \%$ solution of formaldehyde for subsequent histological analysis. The animals were euthanized by overdosing the applied anesthetics.

The resected intestinal segments, after fixation in $10 \%$ formaldehyde solution, were subjected to histological processing for 12 hours in automatic histotechnical. The slides were stained with hematoxylin-eosin and analyzed by optical microscopy (microscope Nikon ${ }^{\circledR}$ E200) by the pathologist without prior knowledge of this on the belonging group of each rat, and were classified according to the degree of tissue lesion according to Chiu et al. ${ }^{17}$ :

- Degree 0: mucosa without changes.

- Grade 1: well-formed villi without cell lysis or inflammatory process, however, with formation of subepithelial space of Grünhagen.

- Grade 2: presence of cell lysis, formation of subepithelial space of Grünhagen and increased spacing between the villi.

- Grade 3: destruction of the free portion of the villi, presence of dilated capillaries, and inflammatory cells.

- Grade 4: structural destruction of the villi, with only a few outline formed by necrotic material and inflammatory cells, hemorrhage and basal glandular ulceration.

- Grade 5: destruction of the entire mucosa, any glandular structure no longer being observed, but only amorphous material deposited on the submucosa.

The results were analyzed statistically, applying the nonparametric Kruskal-Wallis test, with a significance level of $\mathrm{p}<0.05$. It was used the 5.4 Bioestat program.

\section{Results}

After the histological analysis of the degree of damage 
to the intestinal mucosa by Chiu et al. ${ }^{17}$, we found the following results (Table 1 and Figure 2).

TABLE 1 - Results of the histological analysis of the degree of damage to the intestinal mucosa of rats studied.

Rats Grade of mucosa lesion

\begin{tabular}{cccc} 
& Group IR & Group IPC 1 & Group IPC 2 \\
\hline $\mathbf{1}$ & 2 & 1 & 1 \\
$\mathbf{3}$ & 4 & 1 & 3 \\
$\mathbf{4}$ & 3 & 1 & 3 \\
$\mathbf{5}$ & 2 & 3 & 1 \\
$\mathbf{6}$ & 2 & 0 & 1 \\
$\mathbf{7}$ & 3 & 0 & 2 \\
$\mathbf{8}$ & 3 & 1 & 0 \\
$\mathbf{9}$ & 3 & 1 & 1 \\
$\mathbf{1 0}$ & 2 & 3 & 1 \\
Average & 4 & 3 & 1.4
\end{tabular}

Legend: IR: ischemia and reperfusion; IPC: ischemic postconditioning;

Note: $\mathrm{p}<0.05$ between groups $\mathrm{A}$ and $\mathrm{B}$ and between $\mathrm{A}$ and $\mathrm{C} ; \mathrm{p}>0.05$ between groups $\mathrm{B}$ and $\mathrm{C}$

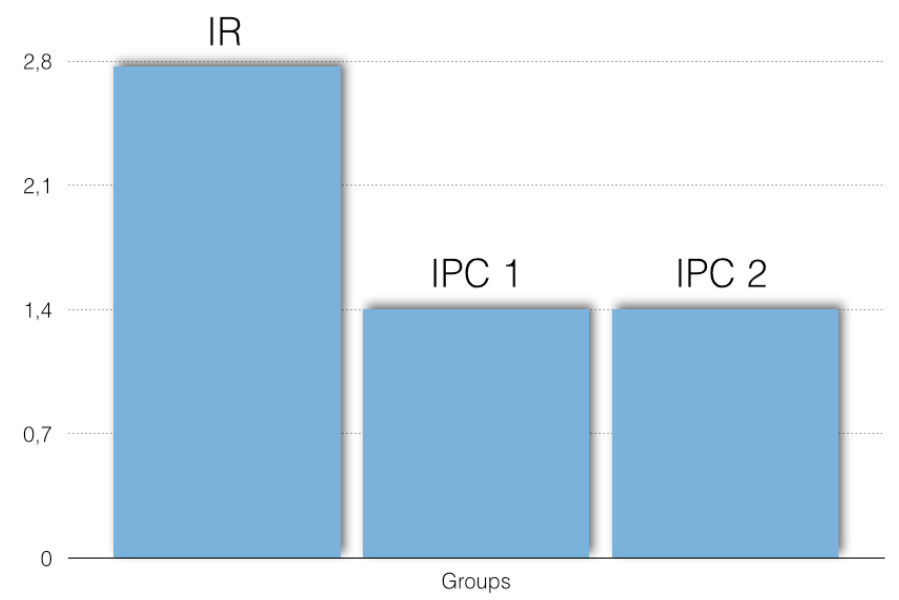

FIGURE 2 - Graphic showing results of the histological analysis of the degree of damage to the intestinal mucosa of rats studied. (IR: ischemia and reperfusion; IPC: ischemic postconditioning);

Note: $\mathrm{p}<0.05$ between groups $\mathrm{A}$ and $\mathrm{B}$ and between $\mathrm{A}$ and $\mathrm{C} ; \mathrm{p}>0.05$ between groups $\mathrm{B}$ and $\mathrm{C}$.
Below the representative photomicrographs of various degrees of intestinal lesion according to Chiu et al. ${ }^{17}$ (Figure 3).

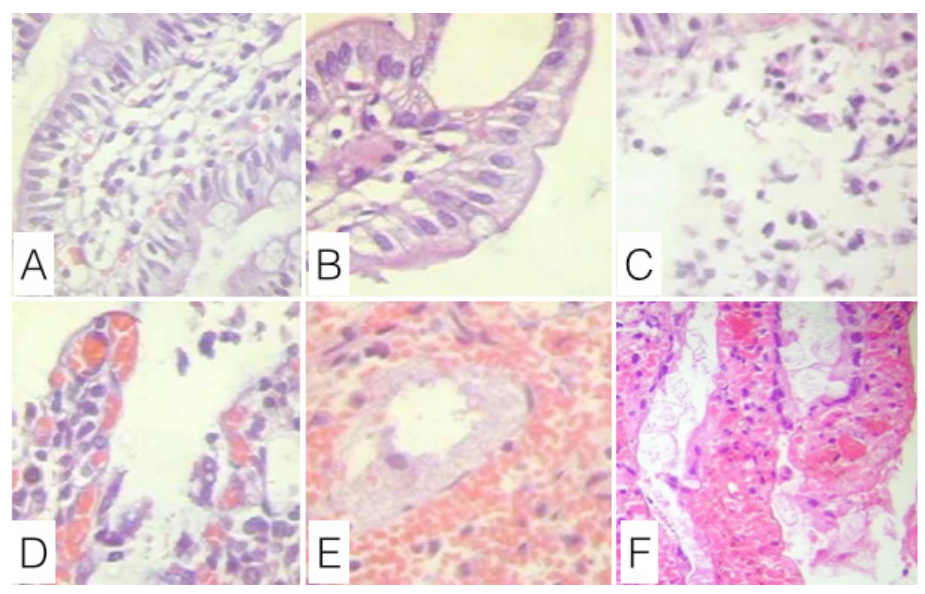

FIGURE 3 - Photomicrographs of various degrees of intestinal lesion according to Chiu et al..$^{17}$ (A-grade 0; B-grade 1; C-grade 2; D-grade 3; E-grade 4; F-grade 5).

\section{Discussion}

The peak of ROS production occurs between the first and seventh minute after initiation of reperfusion, although such substances are detectable in later periods. An abundant production of ROS during this initial phase of reperfusion has been implicated as a major factor in the pathogenesis of tissue injury ${ }^{18}$. The IPC acts at this stage, through a mechanism not fully understood, probably decreasing ROS production by the gradual release of oxygen to cells ${ }^{19}$.

Despite the high number of publications showing good results of IPC, in 2004 Schwartz et al. ${ }^{20}$ demonstrated no benefit in its use in myocardial ischemia and reperfusion in pigs. Also in Bretz et al. ${ }^{15}$ whose aim was to determine whether the IPC could really alleviate the injury caused by ischemia and reperfusion process. They evaluated rabbits $(n=6)$ distributed into control group (1), IR group (2), IR group with IPC. Ischemia was caused by 45 -minute-long by jejunal artery occlusion, followed by two hours of reperfusion. The IPC was performed by four cycles of reperfusion for 30 seconds and reocclusion for 30 seconds too. At the end of the reperfusion, gut segments were collected and the animals euthanized. A semi-quantitative histopathologic evaluation was performed by a single observer and there was no significant difference in the degree of necrosis between the groups ${ }^{10}$.

Nakamura et al. ${ }^{16}$ also showed no benefit in IPC employment using five cycles of reperfusion and ischemia lasting 
30 seconds each, quite like the publication of Bretz et al. ${ }^{15}$, what could permit us to infer that perhaps a greater number of short cycles does not lead to a protective effect with IPC.

In contrasting form, Rosero et al. ${ }^{21}$ recently evaluated three different IPC protocols in rats subjected to mesenteric ischemia and reperfusion, and found that short cycles offered more protection against reperfusion injury. They used 60 minutes of ischemia and 60 minutes of reperfusion, it means that different periods were adopted in the two studies cited above, which may perhaps be the reason for conflicting results.

Sengul et al. ${ }^{10}$ also showed better results with short IPC cycles. They used three and six cycles of IPC lasting 10 seconds each in rats subjected to mesenteric ischemia and reperfusion and obtained better results with six cycles, but both groups had an advantage over the control group.

The results obtained with these publications are conflicting, since the difference between prolonged cycles and shorter cycles is not observed in the present study. It should be noted, however, that the ischemia and reperfusion times used were different from those employed by us compared with the above two articles cited, which could adversely affect the comparison. In this present research, we used two different protocols of PCI. The total period of PCI is equal in both methods, but duration of each one was different. Our results show that there was no difference between two cycles of two minutes or four cycles of 30 seconds, although both have demonstrated efficacy in the prevention of reperfusion lesion. If the total time of PCI is the most important fact to prevent that lesions, its not clear yet and other researches shoul demonstrate it.

Vinten-Johansen et l. $^{22}$ have already suggested in 2005 that in myocardial ischemia and reperfusion, IPC with short cycles have greater efficacy, proposing 30 seconds to dogs and 10 seconds to rats, based on the literature showed that time. We need to do an important consideration here in relation to the studied organ, since what is shown effective for the heart can not be so into the intestine, for example. In addition, other studies with longer times led mostly similar results about the effectiveness of IPC. This study is an example of that, since there was no difference in the ability to minimize reperfusion injury between groups IPC 1 and IPC 2.

Tsang et $a .^{23}$ also demonstrated that in myocardial ischemia and reperfusion, both gradual reperfusion and IPC exerted the same effect, though mechanisms not exactly identical. Therefore, it can be inferred that the IPC by more or less cycles, longer or shorter, can also achieve similar efficacy.

Our results leave us some messages. Firstly, reinforcing the idea that the IPC is really an effective method for the prevention or reduction of reperfusion injury. Second, that the method used here and in rats, there is no difference between making up two cycles of ischemia and reperfusion lasting two minutes or four cycles lasting thirty seconds by H\&E histological evaluation of the ileum after 60-minute reperfusion. Finally, its clear that there are still some points to be clarified as what is the best IPC employment method, and existing conflicting results in the literature on the number of cycles and duration of these may be due to the great diversity of animals studied and especially to ischemia and reperfusion times, which could influence a greater or lesser potential reperfusion injury. This means we must move towards a standardization of development of methods of experimental reperfusion injury to later find out whether there is difference in the effectiveness of IPC with changes of cycles and duration.

\section{Conclusions}

The ischemic postconditioning was able to minimize reperfusion injury of rats undergone mesenteric ischemia and reperfusion process. There was no difference in the effectiveness of the method comparing two cycles of two minutes with four cycles of 30 seconds by H\&E histological evaluation of the ileum after 60-minute reperfusion.

\section{References}

1. Parks DA, Granger DN. Contributions of ischemia and reperfusion to mucosal lesion formation. Am J Physiol. 1986;250:749-53. PMID: 3717337.

2. Zhao ZQ, Corvera JS, Halkos ME, Kerendi F, Wang NP, Guyton RA. Inhibition of myocardial injury by ischemic postconditioning during reperfusion: comparison with ischemic preconditioning. Am J Physiol Heart Circ Physiol. 2003;285(2):579-88. PMID: 12860564.

3. Huang M, Zhang L, Wang Y, Yao J, Weng M, Wu M. Effect of ischemic post-conditioning on spinal cord reperfusion injury in rabbits. Can J Anaesth. 2007;54(1):42-8. PMID: 17197467.

4. Murry CE, Jennings RB, Reimer KA. Preconditioning with ischemia: a delay of lethal cell injury in ischemic myocardium. Circulation. 1986;74:1124-36. PMID: 3769170.

5. Santos CHM, Gomes OM, Pontes JCDV, Miiji LNO, Bispo MAF. Postconditioning: preliminary results of this new option in the treatment of mesenteric ischemia and reperfusion. Cardiovasc Sci Fórum. 2007;2(2):13-24.

6. Santos CHM, Pontes JCDV, Miiji LNO, Nakamura DI, Galhardo CAV, Aguena SM. Postconditioning effect in the hepatic ischemia and reperfusion in rats. Acta Cir Bras. 2010;25(2):163-8. PMID: 20305883

7. Darling EC, Jiang R, Maynard M, P Whittaker, Vinten-Johansen J, Przyklenk K. Postconditioning via stuttering myocardial reperfusion limits infarct size in rabbit hearts: role of ERK1/2. Am J Physiol Heart Circ Physiol. 2005;289(4):618-26. PMID: 15937101.

8. Tang XL, Sato M, Tiwari S, Dawn B, Bi Q, Li Q. Cardioprotection by postconditioning in conscious rats is limited to coronary occlusions 
$<45$ min. Am J Physiol Heart Circ Physiol. 2006;291(5):2308-17. PMID: 16815986

9. Santos CHM, Pontes JCDV, Gomes OM, Miiji LNO, Bispo MAF. Evaluation of ischemic postconditioning effect on mesenteric ischemia treatment. Experimental study in rats. Rev Bras Cir Cardiovasc. 2009;24(2):150-6. PMID: 19768293.

10. Sengul I, Sengul D, Guler O, Hasanoglu A, Urhan MK, Taner AS, Vinten-Johansen J. Postconditioning attenuates acute intestinal ischemia-reperfusion injury. Kaohsiung J Med Sci. 2013;29(3):11927. doi: 10.1016/j.kjms.2012.08.021.

11. Cheng $\mathrm{CH}$, Lin HC, Lai IR, Lai HS. Ischemic postconditioning attenuate reperfusion injury of small intestine: impact of mitochondrial permeability transition. Transplantation. 2013;95(4):559-65. doi: 10.1097/TP.0b013e31827e6b02.

12. Wen SH, Ling YH, Li Y, Li C, Liu JX, Li YS, Yao X, Xia ZQ, Liu $\mathrm{KX}$. Ischemic postconditioning during reperfusion attenuates oxidative stress and intestinal mucosal apoptosis induced by intestinal ischemia/reperfusion via aldose reductase. Surgery. 2013;153(4):555-64. doi: 10.1016/j.surg.2012.09.017.

13. Onody P, Rosero O, Kovács T, Garbaisz D, Hegedüs V, Lotz G, Harsányi L, Szijártó A. Postconditioning - effective method against distant organ dysfunction?. Magy Seb. 2012;65(4):222-9. doi: 10.1556/MaSeb.65.2012.4.9.

14. Rosero O, Onody P, Stangl R, Hegedus V, Lotz G, Blázovics A, Kupcsulik P, Szijártó A. Investigation of postconditioning in intestinal ischemia-reperfusion experimental models. Magy Seb. 2011;64(1):28-36. doi: 10.1556/MaSeb.64.2011.1.6.

15. Bretz B, Blaze C, Parry N, Kudej RK. Ischemic postconditioning does not attenuate ischemia-reperfusion injury of rabbit small intestine. Vet Surg. 2010;39:216-23. PMID: 20210969.

16. Nakamura RK, Santos CHM, Miiji LNO, Arakaki MS, Maedo CM, Ernica-Filho M, Casino PC, Pontes ERJC. Very short cycles of postconditioning have no protective effect against reperfusion injury. Experimental study in rats. Rev Bras Cir Cardiovasc. 2014;29(4):521-6. PMID: 25714204.

17. Chiu CJ, Mcardle AH, Brown R, Scott HJ, Gurd FN. Intestinal mucosal lesion in low-flow states. Arch Surg. 1970;101:478-83. PMID: 5457245.

18. Sun HY, Wang NP, Kerendi F, Halkos M, Kin H, Guyton RA, VintenJohansen J, Zhao ZQ. Hypoxic postconditioning angiograms cardiomyocyte loss by inhibiting ROS generation and intracellular Ca2 + overload. Am J Physiol Heart Circ Physiol. 2005;288:1900-8. PMID: 15563525.
19. Lim SY, Davidson SM, Hausenloy DJ, Yellon DM. Preconditioning and postconditioning: The essential role of the mitochondrial permeability transition pore. Cardiovasc Res. 2007;75(3):530-5. PMID: 17512507.

20. Schwartz ML, Lagranha CJ. Ischemic postconditioning during reperfusion activates Akt and ERK without protecting against lethal myocardial ischemia-reperfusion injury in pigs. Am J Physiol Heart Circ Physiol. 2006 Mar;290(3):H1011-8. PMID: 16214840.

21. Rosero O, Onody P, Stangl R, Turoczi Z, Fulop A, Garbaisz D, Lotz G, Harsanyi L, Szijarto A. Postconditioning of the small intestine: which is the most effective algorithm in a rat model? J Surg Res. 2014;187(2):427-37. PMID: 24238973.

22. Vinten-Johansen J, Yellon DM, Opie LH. Postconditioning: a simple, clinically applicable procedure to improve revascularization in acute myocardial infarction. Circulation. 2005;112:2085-8. PMID: 16203924.

23. Tsang A, Hausenloy DJ, Mocanu MM, Yellon DM. Postconditioning: a form of "modified reperfusion" protects the myocardium by activating the phosphatidylinositol 3-kinase-Akt pathway. Circ Res. 2004;95:230-2. PMID: 15242972.

\section{Correspondence:}

Carlos Henrique Marques dos Santos

Rua Dr Abdalla Duailibi, 264/Lote 11 casa 7

79037230 Campo Grande - MS Brasil

chenriquems@yahoo.com.br

Received: Jun 16, 2015

Review: Aug 18, 2015

Accepted: Sep 17, 2015

Conflict of interest: none

Financial source: none

${ }^{1}$ Research performed at Laboratory of Experimental Surgery, Department of Surgery, Universidade Federal do Mato Grosso do Sul (UFMS), Campo Grande-MS, Brazil. Part of PhD degree thesis, Postgraduate Program in Health and Development of Region Midwest. Tutor: Ricardo Dutra Aydos. 